\title{
SHORT PROOFS OF THEOREMS OF LEKKERKERKER AND BALLIEU
}

\author{
MAX RIEDERLE
}

Eberhardstr. 14

$79 \mathrm{Ulm} /$ Donau

West Germany

(Received October 16, 1981)

ABSTRACT. For any irrational number $\xi$ let $A(\xi)$ denote the set of all accumulation points of $\{z: \quad z=q(q \xi-p), p \in \mathbb{Z}, q \in \mathbb{Z}-\{0\}, p$ and $q$ relatively prime $\}$. In this paper the following theorem of Lekkerkerker is proved in a short and elementary way: The set $A(\xi)$ is discrete and does not contain zero if and only if $\xi$ is a quadratic irrational. The procedure used for this proof simultaneously takes care of a theorem of Ballieu.

KEY WORDS AND PHRASES. Lekkerkerker's Theorem, Approximation of numbers, Cuadratic Irrationals. 1980 MATHEMATICS SUBJECT CLASSIFICATION CODES. $10 F 05,10 F 35$.

1. INTRODUCTION.

This paper is easily readable by anyone familiar with the elements of continued fractions, as far as Lagrange's theorem on the periodic representation of quadratic irrationals. Throughout this paper, $\xi$ denotes an irrational number wich is represented by the regular continued fraction $\xi=\left[b_{0}, b_{1}, b_{2}, \ldots\right]=\left[b_{0}, b_{1}, \ldots, b_{n-1}, \xi_{n}\right]$ and $A(\xi)$ stands for the set of all the real accumulation points of $\{z: z=q(q \xi-p)$, $\mathrm{p} \in \mathbb{Z}, \mathrm{q} \in \mathbb{Z}-\{0\}, \mathrm{p}$ and $\mathrm{q}$ relatively prime $\}$. Obvious $1 \mathrm{y}, \mathrm{A}(\xi)$ describes those Dirichlet approximation qualities which occur infinitely often. Furthermore, for any sequence $\left(a_{n}\right)$ let $H\left(a_{n}\right)$ denote the set of all its limit points and for $x \in \mathbb{R}$ and $\varepsilon>0$ set $B(x, \varepsilon)=(x-\varepsilon, x+\varepsilon)$. The main purpose of this paper is to give a simple proof of the following theorem of Lekkerkerker [1] (cf. also [2])・ 
The set $A(\xi)$ is discrete and does not contain zero if and only if $\xi$ is a quadratic irrational.

The proof of the sufficient part of the theorem mainly depends on the irreducible polynomial of $\xi$, whereas the necessary part is a consequence of the relation between $A(\xi)$ and the sequence $\left(\xi_{n}\right)$ and simultaneously establishes the following theorem of Ballieu [3]:

The set $H\left(\xi_{n}\right)$ is finite and $\left(\xi_{n}\right)$ is bounded if anj only if $\xi$ is a quadratic irrational.

Finally for any quadratic irrational $\xi$ we shall show how to evaluate $A(\xi)$ in an easy way.

\section{BASIC FORMULAS.}

In this section we state the formulas used in the sequel. Let $A_{n} / B_{n}$ denote the $n$-th convergent of $\left[b_{0}, b_{1}, b_{2}, \ldots\right]$ where $A_{n}$ and $B_{n}$ are relatively prime. Set $\rho_{n}=B_{n} / B_{n-1}$ and put $\delta_{n}=B_{n}\left(B_{n} \xi-A_{n}\right)$. Then the following formulas hold for all $\mathbf{n} \in \mathbf{m}_{0}:$

$$
\begin{aligned}
& \delta_{n}=\frac{(-1)^{n}}{\xi_{n+1}+1 / \rho_{n}} \\
& \delta_{n-1}=\frac{(-1)^{n-1}}{\rho_{n}+1 / \xi_{n+1}} \\
& \xi_{n+1}=\frac{1+\sqrt{1+4 \delta_{n} \delta_{n-1}}}{2 \delta_{n}}(-1)^{n} .
\end{aligned}
$$

PROOF. Equation (2.1) is an easy consequence of the well known identity $\xi-A_{n} / B_{n}=(-1)^{n} /\left(B_{n}\left(B_{n} \xi_{n+1}+B_{n-1}\right)\right)$, formula (2.2) can be derived from (2.1) when using the identities $\xi_{n}=b_{n}+1 / \xi_{n+1}$ and $\rho_{n}=b_{n}+1 / \rho_{n-1}$ and, finally, (2.3) can be obtained when combining (2.1) and (2.2).

\section{PROOF OF LEKKERKERKER'S THEOREM.}

(i) Suppose $\xi$ is a quadratic irrational. There exists an indefinite quadratic form $f(x, y)=a x^{2}+b x y+c y^{2}$ with $a, b, c \in \mathbb{Z}$ and $f(\xi, 1)=0$. If $\zeta$ denotes the algebraic conjugate of $\xi$ then if follows by Vieta's theorem that

$$
f(p, q)=a(p-q \xi)(p-q \zeta)=a q(q \xi-p)(\zeta-p / q)
$$

for all $(p, q) \in \mathbb{Z} \times(\mathbb{Z}-\{0\})$ which implies that 


$$
q(q \xi-p)=\frac{f(p, q)}{a(\zeta-p / q)}
$$

When using the notation $\tilde{z}=\{(x, y): x, y \in \mathbb{Z}, y \neq 0, x$ and $y$ relatively prime $\}$ equation (3.2) implies that

$$
A(\xi) \subset \frac{f(\tilde{Z})}{a(\zeta-\xi)},
$$

since if $\left(p_{n}, q_{n}\right) \in \tilde{z}$ with ${ }_{n} l i m_{\infty} q_{n}\left(q_{n} \xi-p\right) \in A(\xi)$ then ${ }_{n} 1 \dot{j} m_{\infty} p_{n} / q_{n}=\xi$. Clearly, $f(Z) \subset \mathbb{Z}$ and we conclude that $A(\xi)$ is discrete. Now from (3.1) we can see that $f(p, q) \neq 0$ for all $(p, q) \in \tilde{Z}$ and hence it follows by (3.3) that $0 \notin A(\xi)$.

(ii) Suppose that $A(\xi)$ is discrete and $0 \notin A(\xi)$. From equation (2.1) we can see that $\left|\delta_{n}\right| \leq 1$ for all $n \in \mathbb{N}_{0}$. Therefore and since all the numbers $\delta_{n}$ are distinct, $H\left(\delta_{n}\right)$ is a compact subset of $A(\xi)$ and hence $H\left(\delta_{n}\right)$ is finite and $0 \notin H\left(\delta_{n}\right)$. Now by (2.3) it is easy to see that $H\left(\xi_{n}\right)$ is finite and $\left(\xi_{n}\right)$ is bounded. Therefore, in order to complete the proof, it suffices to prove Ballieu's theorem.

\section{PROOF OF BALLIEU'S THEOREM.}

(i) Suppose that $\left(\xi_{n}\right)$ is bounded and $H\left(\xi_{n}\right)$ is finite, say $H\left(\xi_{n}\right)=\left\{z_{1}, \ldots, z_{m}\right\}$. It follows from the identity $\xi_{n}=\left[b_{n}, b_{n+1}, \ldots\right]$ that there exists a $k \in \mathbf{N}$ such that $b_{n} \leq k$ for all $n \in \mathbb{N}$, and hence $b_{n}+1 / k \leq \xi_{n} \leq b_{n}+1-1 /(k+1)$. Therefore, the set $H\left(\xi_{n}\right) \cap z$ is empty and we can find a number $\varepsilon>0$ such that the sets $B\left(z_{\nu}, \varepsilon\right)$ are pairwise disjoint and contained in $\mathbb{R}-\mathbf{z}$.

Let I(z) denote the greatest integer not exceeding $z$ and for $z \notin z$ put $\tau(z)=(z-I(z))^{-1}$. Clearly, $\xi_{n+1}=\tau\left(\xi_{n}\right)$ for all $n \in \mathbb{N}_{0}$. Also the function $\tau$ is continuous on $H\left(\xi_{n}\right)$, therefore $\tau\left(H\left(\xi_{n}\right)\right) \subset H\left(\xi_{n}\right)$ and we can find a $\delta, 0<\delta<\varepsilon$, such that $\tau\left(B\left(z_{v}, \delta\right)\right) \subset B\left(\tau\left(z_{v}\right), \varepsilon\right)$ for all $v \in\{1, \ldots, m\}$. There exists a number $n_{0}$ such that $\xi_{n_{0}} \in B\left(z_{1}, \delta\right)$ and $\xi_{n} \in \bigcup_{\nu=1}^{m} B\left(z_{v}, \delta\right)$ for all $n \geq n_{0}$. Therefore, when writing $\tau^{(p)}$ for the p-th composition map of $\tau$, we obtain by induction that $\xi_{n_{0}}+p \in B\left(\tau^{(p)}\left(z_{1}\right), \delta\right)$ for all $p \in \mathbb{N}_{0}$. Since $\tau\left(H\left(\xi_{n}\right)\right) \subset H\left(\xi_{n}\right)$ and $H\left(\xi_{n}\right)$ is finite, the sequence $\left(\tau^{(p)}\left(z_{1}\right)\right), p \in N_{0}$, is periodic. From the identities $b_{n_{0}}=I\left(\xi_{n_{0}}+p\right)$ $=I\left(\tau^{(p)}\left(z_{1}\right)\right)$ we conclude that the sequence $\left(b_{n_{0}}+p\right), p \in \mathbb{N}_{0}$, is periodic and thus, by Lagrange's theorem, $\xi$ is a quadratic irrational.

(ii) The other direction of Ballieu's theorem is an easy consequence of 
Lagrange's theorem.

5. CONCLUDING REMARKS.

The inclusion in (3.3) is actually an equality. In order to prove this, we need the following well known theorem (cf. [4], p. 22-23):

Let $f(x, y)$ be an indefinite quadratic form with integer coefficients and let $\xi$ be one of its roots. Then for any pair $(p, q) \in \mathbb{Z} \times(\mathbb{Z}-\{0\})$ there are infinitely many relatively prime integers $p_{n}, q_{n}$ such that $f\left(p_{n}, q_{n}\right)=f(p, q)$ for all $n \in \mathbb{N}$ and $\mathrm{n}^{1} \operatorname{im}_{\infty}\left(\mathrm{q}_{\mathrm{n}} \xi-\mathrm{p}\right)=0$.

In fact, this result combined with (5) and (6), leads to the following:

THEOREM. Suppose that $\xi$ is a quadratic irrational, say $f(\xi, 1)=0$ for some indefinite quadratic form $f(x, y)$ with integer coefficients. Moreover, let $\zeta$ be the algebraic conjugate of $\xi$. Then

$$
A(\xi)=\frac{f(\tilde{Z})}{f(1,0)(\zeta-\xi)} \text {. }
$$

\section{REFERENCES}

1. LEKKERKERKER, C.G. Una questione di approssimazione diofantea e una proprieta caratteristica dei numeri quadratici I, II. Atti Accad. Naz. Lincei. Rend. C1. Sci. Fis. Mat. Nat. 21 (1956) 179-185, 257-262.

2. JURKAT, W.B. and PERERIMHOFF, A. Characteristic approximation properties of quadratic irrationals, Intern. J. of Math. and Math. Sci. I (1978) 479-496.

3. BALLIEU, R. Sur des suites de nombres liées à une fraction continue régulière, Acad. Roy. Belg. Bull. C1. Sci. 29 (1943) 165-174.

4. CASSELS, J.W.S. An Introduction to Diophantine Approximation. Cambridge Univ. Press, Cambridge, 1965.

5. PERRON, 0. Die Lehre von den Kettenbrüchen. Teubner Verlag, Stuttgart, 1954. 


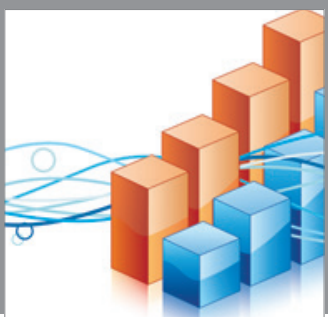

Advances in

Operations Research

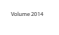

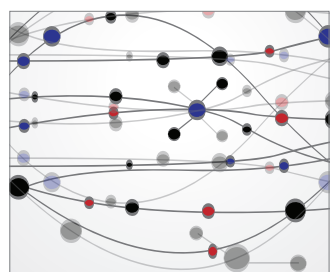

\section{The Scientific} World Journal
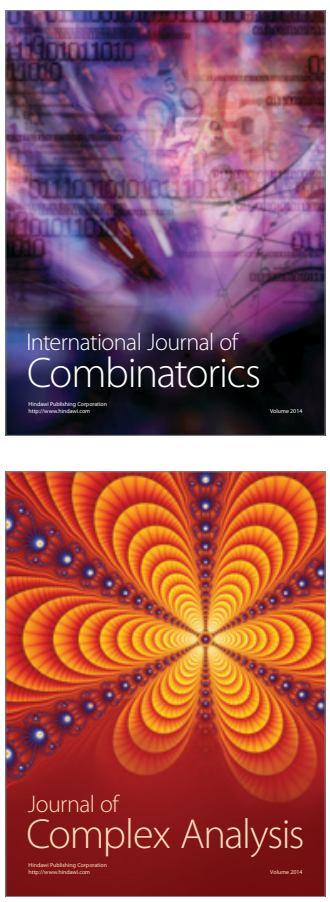

International Journal of

Mathematics and

Mathematical

Sciences
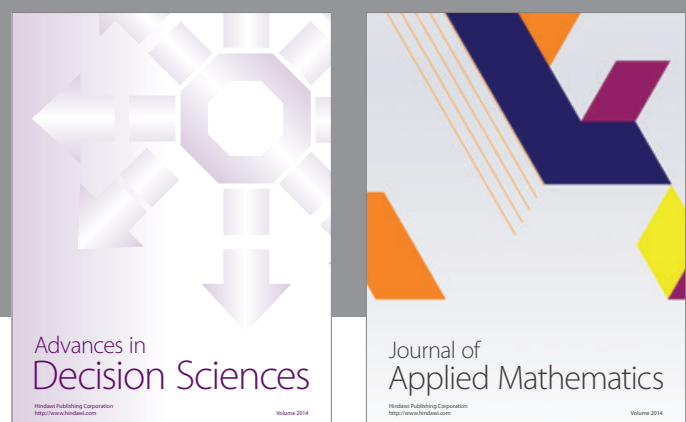

Journal of

Applied Mathematics
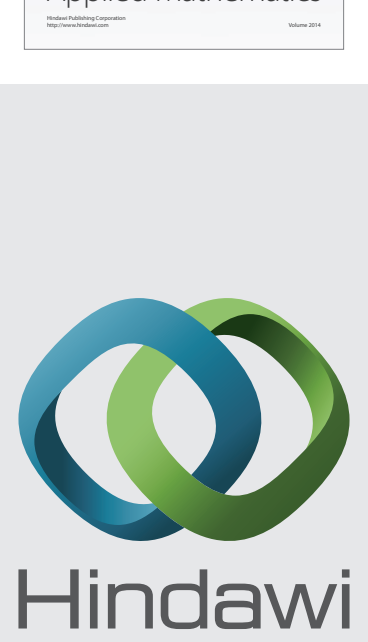

Submit your manuscripts at http://www.hindawi.com
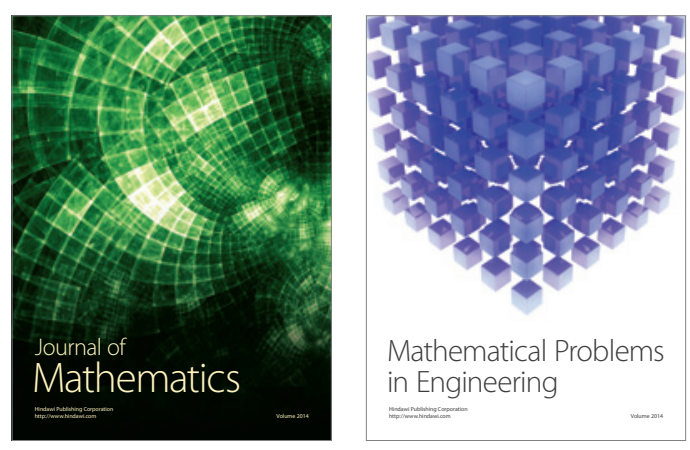

Mathematical Problems in Engineering
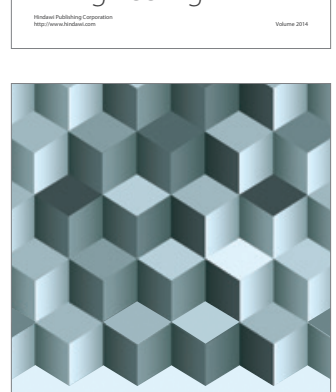

Journal of

Function Spaces
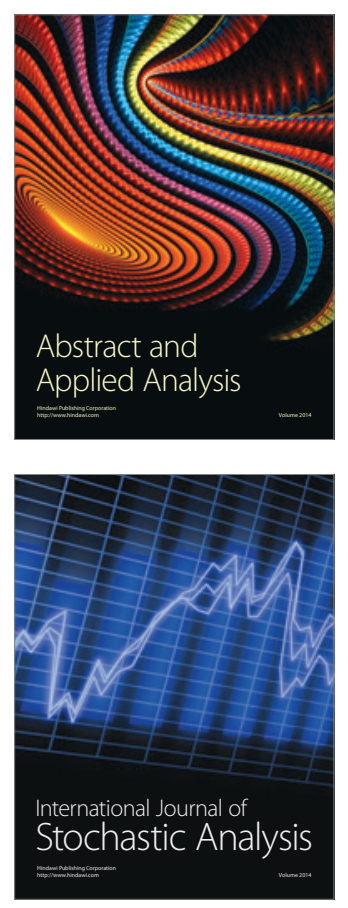

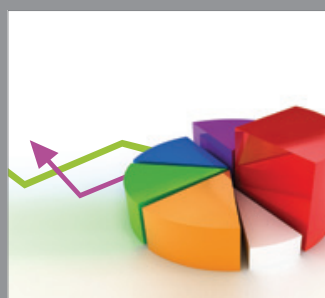

ournal of

Probability and Statistics

Promensencen
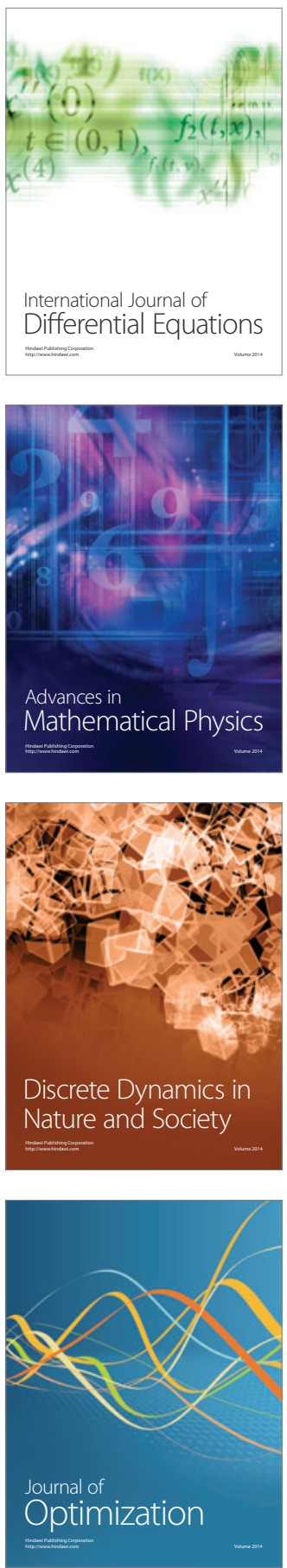